\title{
Improve The Effective Resonance Light Scattering Of Three-Layered Plasmonic Au@Dielectric@Ag Bimetallic Nanoshells
}

Jian Zhu ( $\sim$ nanoptzj@163.com )

Xi'an Jiaotong University https://orcid.org/0000-0002-4075-6991

Shu-min Zhao

Xi'an Jiaotong University

\section{Research Article}

Keywords: surface plasmon resonance (SPR), bimetallic, three-layered nanoshells, effective light scattering, local dielectric environment

Posted Date: June 7th, 2021

DOI: https://doi.org/10.21203/rs.3.rs-527754/v1

License: (c) (i) This work is licensed under a Creative Commons Attribution 4.0 International License.

Read Full License

Version of Record: A version of this preprint was published at Plasmonics on August 2nd, 2021. See the published version at https://doi.org/10.1007/s11468-021-01510-w. 


\title{
Improve the effective resonance light scattering of three-layered plasmonicAu@Dielectric@Ag bimetallic nanoshells
}

\author{
Jian Zhu ${ }^{a *}$, Shu-min Zhao ${ }^{b}$ \\ ${ }^{a}$ The Key Laboratory of Biomedical Information Engineering of Ministry of Education, School of \\ Life Science and Technology, Xi'an Jiaotong University, Xi'an 710049, China \\ ${ }^{b}$ Non-equilibrium Condensed Matter and Quantum Engineering Laboratory, School of Science, \\ Xi'an Jiaotong University, Xi'an 710049, China \\ * Authors to whom correspondence should be addressed. \\ Electronic mail: nanoptzj@163.com \\ Tel.: 86-29-82664224. \\ Address: School of Life Science and Technology, \\ Xi'an Jiaotong University, \\ Xi' an, 710049, \\ Peoples Republic of China
}




\begin{abstract}
:
The strong light scattering from SPR has received an extraordinary attention due to the useful applications in photodetectors, cell and biomedical imaging. However, the applications using light scattering require a high scattering cross-section along with low absorption losses near the resonance wavelength. In this paper, effective plasmonic scattering of three-layered $\mathrm{Au}-\mathrm{Ag}$ bimetallic nanoshells with a dielectric separate layer has been studied using the quasi-static approximation of classical electrodynamics. Because of the surface plasmon resonance (SPR)-induced intense light absorption, the effective scattering intensity is much weaker than that of scattering cross section. However, the effective scattering intensity could be improved by tuning the geometric dimension and local dielectric environment of the nanostructure. It has been found that the greatest effective scattering takes place when the outer Ag nanoshell has a thick thickness or the dielectric separate layer has a small dielectric constant. The effective scattering also depends on the inner Au sphere radius and outer surrounding dielectric constant. Because of the mode transformation of the SPR, the effective scattering could also be greatly improved when the inner Au sphere has a very small or large size. However, the effective scattering intensity changes non-monotonously as the surrounding dielectric constant increases. The greatest effective scattering could be obtained when the surrounding dielectric constant has an intermediate value. This tunable effective plasmonic scattering of Au@Ag three-layered nanoshells presents a potential for design and fabrication of plasmonic optical nanodevice based on resonance light scattering.
\end{abstract}

Key words: surface plasmon resonance (SPR); bimetallic; three-layered nanoshells; effective light scattering; local dielectric environment 


\section{Introduction}

The plasmonic optical properties of gold and silver nanoparticles with core-shell structure have been studied extensively in literature. When the surface plasmon resonance (SPR) has been excited, the collective motion of the surface free electrons oscillates like a simple dipole and consequently results in strong light absorption and scattering, the sum of the absorption and scattering is defined as extinction. The strong light scattering from SPR has received an extraordinary attention due to the useful applications in photodetectors, cell and biomedical imaging etc. [1-9]. However, both light absorption and scattering are all resulted from SPR, thus they have similar resonance wavelength. Therefore, the light scattering will be greatly reduced by the light absorption. So the applications based on light scattering require a high scattering cross-section along with low absorption losses [10]. In order to express the contribution of scattering among the extinction, scattering quantum yield [11], optical radiation efficiency [12] or scattering efficiency [13] has been used and has been defined as the ratio of the scattering cross section $\left(\mathrm{Q}_{\mathrm{sca}}\right)$ to the total extinction cross section $\left(\mathrm{Q}_{\mathrm{ext}}\right)$ at the resonance frequency, scattering efficiency $\eta=\left.\frac{Q_{s c a}}{Q_{\text {ext }}}\right|_{\text {Res }}$.

Because the properties of SPR greatly depend on the particle shape, structure and the local dielectric environmental refractive index, the scattering efficiency could also been fine tuned. Lee et al. reported the dependence of the enhanced scattering efficiency of gold nanorods on aspect ratio, size, end-cap shape, and medium refractive index [11]. They found that the relative contribution of scattering to total extinction is increased as the aspect ratio becomes slightly above 1 for particles having the same number of gold atoms. What's more, the scattering efficiency could be further enhanced as the particle shape changes from a spheroid to a cylindrical geometry. Park et al. studied the optical extinction, absorption, scattering, and their relative contributions of gold nanorods across a wide range of volume and aspect ratio [13]. It has been found that the relative enhancement of scattering efficiency gets intense with the increasing aspect ratio, but saturates at intermediate values. Based on Mie theory, scattering efficiencies of $\mathrm{Ag}-\mathrm{Cu}, \mathrm{Ag}-\mathrm{Au}$, and $\mathrm{Au}-\mathrm{Cu}$ alloy nanoparticles have been studied by Bansal et al. [14]. They found that the enhancement of scattering efficiency could be tuned with both the alloy composition and the surrounding dielectric medium. Bansal et al. also reported the scattering and absorption efficiencies of semiconductor-coated gold nanoshell using the extended Mie theory [15]. They found that both the scattering and absorption efficiencies blue shift with an increase of gold shell layer thickness and the increase of surrounding dielectric constant, whereas the corresponding SPR peaks shift towards longer wavelength. Therefore, the scattering efficiency peak does not always have the same wavelength of the 
SPR. The wavelength drift between resonance light absorption and scattering of plasmonic nanoparticle has also been reported [10]. Both the red and blue drifts of the scattering peak from the absorption peak have been observed in gold nanosphere, nanoshell and nanorod. The wavelength drift between the light absorption and scattering could be further optimized by tuning the particle shape and structure. Therefore, the greatest scattering efficiency may locate at a different wavelength from the SPR. Then, how to find the real peak of the light scattering intensity? In this paper, a new physical quantity denoted as effective scattering has been used to study the intensity of the real light scattering intensity.

On the other hand, optical properties of multilayered metal nanoshells have attracted much attention recently because of the plasmon coupling between inner metal core and outer metal shell [16-23]. In addition, the dielectric constant of the separate layer and outer surrounding also greatly affect the optical properties of the multilayered nanoshells $[16,18]$. Wu et al. reported the effect of dielectric core and embedding medium on the optical properties of gold nanoshells [24]. They found that the increase of the core dielectric constant reduces the strengths of dipole and quadrupole modes. As the surrounding dielectric constant is increased, the quadrupole mode amplitude gets intense, whereas the dipole mode amplitude increases first and then decreases. The effect of the local dielectric environment on the plasmon coupling in $\mathrm{Al}-\mathrm{Au}$ bimetallic metal-dielectric-metal nanoshells has also been studied [25]. It has been found the absorbance changes between different plasmon modes decrease as the separate layer dielectric constant is increased, but increase as the outer environmental dielectric constant is increased. The local dielectric environment also greatly affects the local electric field enhancement in double concentric silver nanotubes [26]. In the inner dielectric core, the major local field factor peak usually changes nonmonotonously as the dielectric constant of inner core or spacer layer is increased. In the dielectric spacer layer, the intense local field could be obtained with small dielectric constant of the spacer layer and reaches the maximum value when the double tube has thick thickness of inner and outer tube. The effect of dielectric coating on the local electric field enhancement of Au-Ag core-shell nanostructures has also been reported [27]. It has been found the antibonding plasmon-induced local field peak always fades down as the dielectric coating thickness is increased. Whereas for bonding plasmon mode, the dielectric coating thickness-dependent monotonic intensity decrease of the local field peak only takes place when the Au-Ag nanoshell has a very small or a very large gold core. Then how does the effective scattering of the bimetallic three-layered nanoshells depend on the local dielectric environment? In this paper, effective plasmonic scattering of Au-Ag bimetallic three-layered nanoshells has been studied theoretically. It has been found the effective scattering greatly depends on the geometric dimension and local dielectric environment of the nanostructure. It has been found that the greatest effective 
scattering could be obtained when the outer Ag nanoshell has a thick thickness or the dielectric separate layer has a small dielectric constant. The effective scattering could also be greatly improved when the inner Au sphere has a very small or large radius.

\section{The Modeling}

The Au@Ag bimetallic three-layered nanostructure with concentric symmetry used in this study is modeled as a dielectric layer-coated gold nanosphere surrounded by a silver nanoshell [25]. In this model, the inner Au nanosphere has a radius of $r_{l}$ and dielectric constant of $\varepsilon_{1}$, the separate dielectric nanolayer has a thickness of $r_{2}-r_{1}$ and dielectric constant of $\varepsilon_{2}$, the outer Ag nanoshell has a thickness of $r_{3}-r_{2}$ and dielectric constant of $\varepsilon_{3}$, the surrounding media has a dielectric constant of $\varepsilon_{4}$. In this study, the dielectric properties of the inner gold sphere and outer silver shell are calculated using a Drude model, $\varepsilon(\omega)=\varepsilon_{b}-\frac{\frac{\omega_{p}^{2}}{\omega^{2}}}{1+\frac{1}{\omega^{2} \tau^{2}}}+i \frac{\frac{\omega_{p}^{2}}{\omega^{2}}}{\omega \tau\left(1+\frac{1}{\omega^{2} \tau^{2}}\right)}$, where $\varepsilon_{b}$ is the bulk metal dielectric constant of gold or silver, $\omega_{p}$ is the bulk metal plasmon frequency of gold or silver, and $\tau$ is the relaxation time [28]. In this calculation, the total diameter of the nanoparticle is set as $50 \mathrm{~nm}$, which is much smaller than the wavelength of the incident light. Therefore, the quasi-static theory can be employed in the calculation of the wavelength-dependent absorption cross section $Q_{a b s}$ and scattering cross section $Q_{s c a}$ of the Au-dielectric-Ag three-layered nanoshells. The detail analytical equations of the wavelength-dependent absorption and scattering cross sections could be derived from the Laplace's equation and could be found in the Ref. [29-31]. At last, the effective scattering cold be obtained as $Q_{s c a} \cdot \frac{Q_{s c a}}{Q_{a b s}+Q_{s c a}}$.

\section{Results and discussions}

\subsection{The spectrum comparison of absorption, scattering and scattering efficiency}

Figure 1a compared the spectra of light absorption, scattering and scattering efficiency. In this calculation, the Au@Ag three-layered nanoshell has a geometrical parameter of $r_{1,2,3}=[10,20,25] \mathrm{nm}$. The dielectric constants of the middle separate layer and the outer surrounding medium are set as $\varepsilon_{2}=5$ and $\varepsilon_{4}=1$, respectively. The absorption spectrum shows three distinct SPR peaks at $403 \mathrm{~nm}, 588 \mathrm{~nm}$ and $823 \mathrm{~nm}$, respectively. The first absorption peak at $403 \mathrm{~nm}$, denoted as $\left|\omega_{+}^{-}\right\rangle$, is from the symmetric coupling between the inner sphere plasmon and the outer anti-bonding shell plasmon [32]. The second peak at $588 \mathrm{~nm}$ is attributed to the symmetric coupling 
between the inner sphere plasmon and the outer bonding shell plasmon, which is denoted as $\left|\omega_{-}^{+}\right\rangle$. The third peak at $823 \mathrm{~nm}$, denoted as $\left|\omega_{-}^{-}\right\rangle$, is from the anti-symmetric coupling between the inner sphere plasmon and the outer bonding shell plasmon. In Figure 1a, the scattering spectrum also exhibits three peaks. Although the wavelength positions of these three scattering peaks are around the frequencies of SPR, the scattering intensity is much weaker than that of absorption. Therefore, we also plotted the spectrum of scattering efficiency, $\eta=\frac{Q_{s c a}}{Q_{a b s}+Q_{s c a}}$, in Figure 1a. One can also find three scattering efficiency peaks, whereas the peak intensities of the scattering efficiency are smaller than 0.1 . What's more, the scattering efficiency peaks get wider and red shift to $434 \mathrm{~nm}, 645 \mathrm{~nm}$ and $1200 \mathrm{~nm}$, respectively. Therefore, the peak wavelengths of the scattering efficiency do not have the same resonance wavelength of the plasmonic absorption and scattering peaks. In order to find the real intensity of the light scattering, a new physical quantity, denoted as effective scattering $=Q_{s c a} \cdot \frac{Q_{s c a}}{Q_{a b s}+Q_{s c a}}$, has been used in this study. In Figure 1b, the spectra of scattering and effective scattering have been compared. One can find the maximum effective scattering takes place around the SPR frequencies. However, the intensity of the effective scattering is much weaker than the scattering cross section. Therefore, improving the effective scattering by tuning the particle dimension and local dielectric environment is very important, which will be discussed detailedly in the next section.

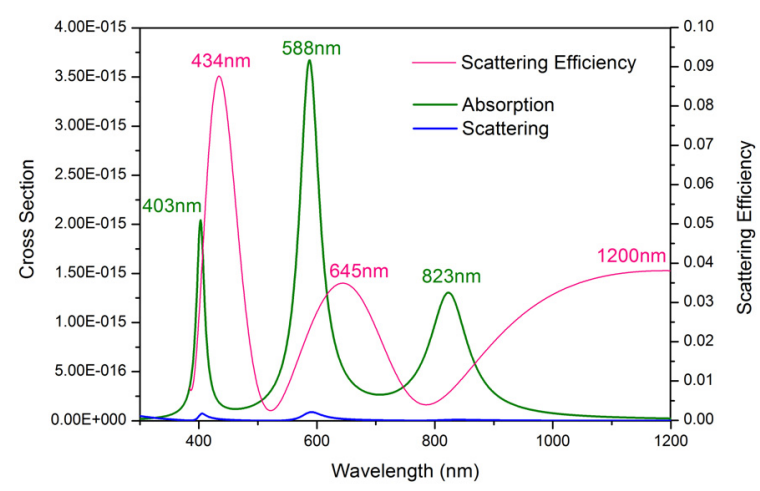

(a)

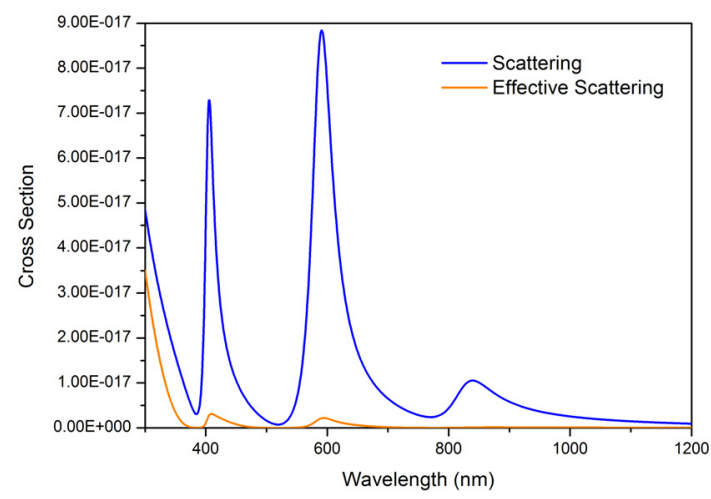

(b)

Figure 1 (a) The spectrum of light absorption, scattering and scattering efficiency. (b) The spectral comparison between scattering and effective scattering. $\mathrm{r}_{1,2,3}=[10,20,25] \mathrm{nm}, \varepsilon_{2,4}=[5,1]$. 
3.2.1 The effect of separate layer dielectric constant on the effective scattering

Because the polarizability of the separate layer affects the collective oscillation strength of the conducting electrons of the nanoshell, the effective scattering of the three-layered nanostructure also depends on the dielectric constant of the separate nanolayer. Figure 2 summarized the effects of $\varepsilon_{2}$ on the effective scattering spectra of Au-dielectric-Ag nanoshells with different inner Au core size and outer dielectric environment. In this calculation, the outer silver nanoshell has a thickness of $5 \mathrm{~nm}$. When the inner gold sphere has a small radius of $5 \mathrm{~nm}$, only one effective scattering peak corresponding to the $\left|\omega_{-}^{-}\right\rangle$mode takes place in the spectra. What's more, the effective scattering peak always decreases quickly and red shifts slightly as the dielectric constant of the separate layer is increased, as shown in Figure 2a,b. In Figure 2b, the dielectric constant of the outer surrounding is decreased to $\varepsilon_{4}=2$, which leads to the effective scattering peak has a shorter wavelength position and the effective scattering has a greater intensity. When the inner gold sphere has a large radius of $15 \mathrm{~nm}$, all the three effective scattering peaks could be observed in the spectra. But only the $\left|\omega_{-}^{+}\right\rangle$mode takes the major role in the spectra, as shown in Figure 2c,d. As the dielectric constant of the separate layer is increased from 1 to 6 , the effective scattering peak corresponding to the $\left|\omega_{-}^{-}\right\rangle$mode still decreases quickly and red shifts, which is similar to the results of Figure 2a,b. In Figure 2c,d, the effective scattering peak corresponding to the $\left|\omega_{+}^{-}\right\rangle$mode could also be observed, but the intensity is too weak. When the dielectric constant of the outer surrounding medium has a large value of $\varepsilon_{4}=5$, the effective scattering peak corresponding to the $\left|\omega_{-}^{+}\right\rangle$mode gets intense distinctly as the $\varepsilon_{2}$ is increased, as shown in Figure 2c. Whereas when the surrounding dielectric constant is decreased to $\varepsilon_{4}=2$, the $\varepsilon_{2}$-dependent effective scattering intensity becomes non-monotonous. The increase of the separate layer dielectric constant leads to the effective scattering intensity increases firstly and then decreases, as shown in Figure 2d.

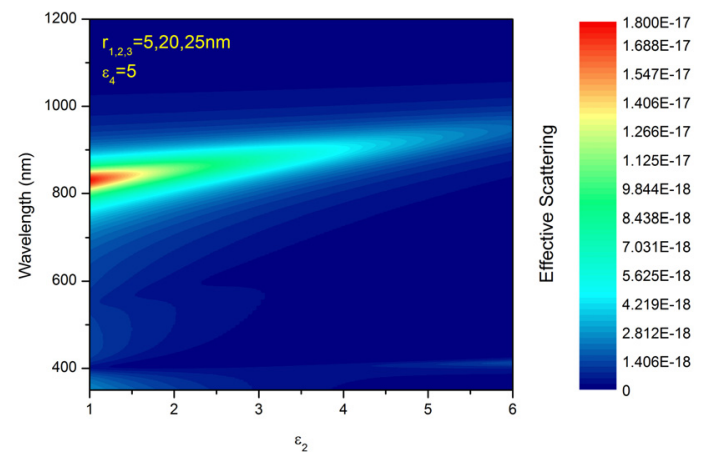

(a)

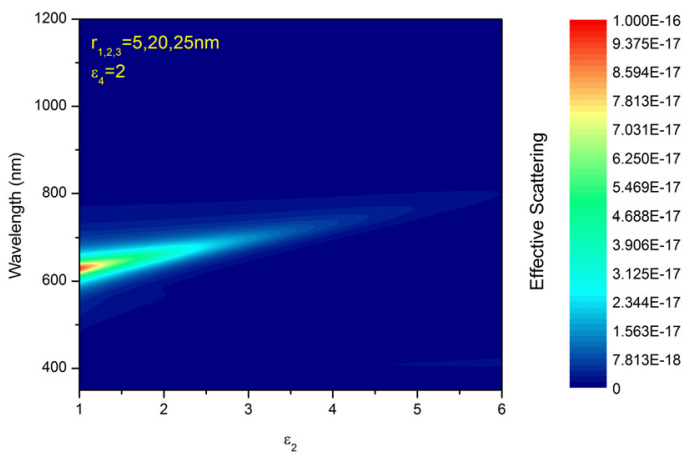

(b) 


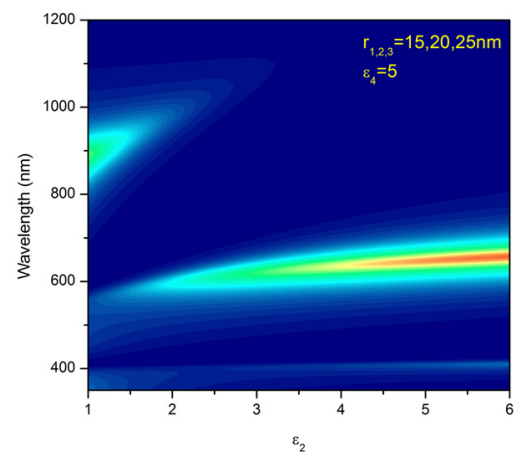

(c)

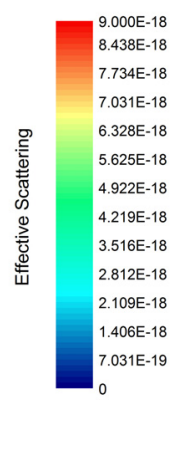

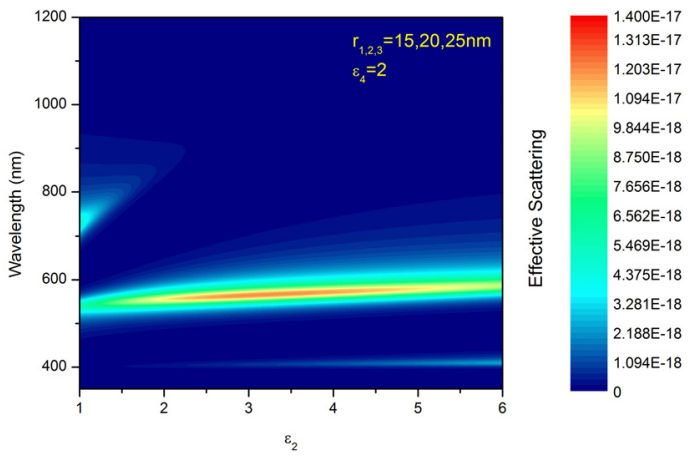

(d)

Figure 2 The effect of $\varepsilon_{2}$ on the effective scattering spectra of Au-Di-Ag nanoshells. (a) with a small inner gold nanosphere and large environmental dielectric constant, (b) with a small inner gold nanosphere and small environmental dielectric constant, (c) with a large inner gold nanosphere and large environmental dielectric constant, (d) with a large inner gold nanosphere and small environmental dielectric constant.

\subsubsection{The effect of surrounding dielectric constant on the effective scattering}

The dielectric constant of the surrounding environment also impacts the effective scattering of the $\mathrm{Au} @ \mathrm{Ag}$ bimetal three-layered nanostructure. The effect of $\varepsilon_{4}$ on the effective scattering spectra of Au-dielectric-Ag nanoshells with different inner core size and separate layer dielectric constant is shown in Figure 3. In this calculation, the thickness of the outer silver nanoshell is still set as $5 \mathrm{~nm}$. When the inner gold sphere has a small radius of $5 \mathrm{~nm}$, the effective scattering peak corresponding to the $\left|\omega_{-}^{-}\right\rangle$mode takes the major role in the spectra, and changes non-monotonously as the surrounding dielectric constant is increased, as shown in Figure 3a,b. The increase of the $\varepsilon_{4}$ always leads to the effective scattering increases firstly and then decreases. When $\varepsilon_{2}=5$, the maximum effective scattering takes place when $\varepsilon_{4}=3.3$. Whereas when the separate layer dielectric constant has a smaller value of $\varepsilon_{2}=2$, the maximum effective scattering has a larger value and appears when $\varepsilon_{4}=2$. When the inner gold sphere has a large radius of $r_{l}=15 \mathrm{~nm}$, the effective scattering peak corresponding to the $\left|\omega_{-}^{+}\right\rangle$mode takes the major role in the spectra, but the $\left|\omega_{-}^{-}\right\rangle$mode becomes very weak, as shown in Figure 3c,d. One can find the $\varepsilon_{4}$-dependent red shift of the effective scattering peak becomes very weak. However, the increase of the $\varepsilon_{4}$ also always leads to the effective scattering increases firstly and then decreases. And the maximum effective scattering also left shifts to a small value of $\varepsilon_{4}$, which is similar to the results in Figure 3a,b. This non-monotonous change of 
the effective scattering could be attributed to the competition between the dielectric constants of separate layer and outer surrounding. The different polarization directions of separate layer and outer surrounding result in opposite contributions to the restoring force and SPR. So the maximum effective scattering always appears when $\varepsilon_{2}$ is close to $\varepsilon_{4}$.

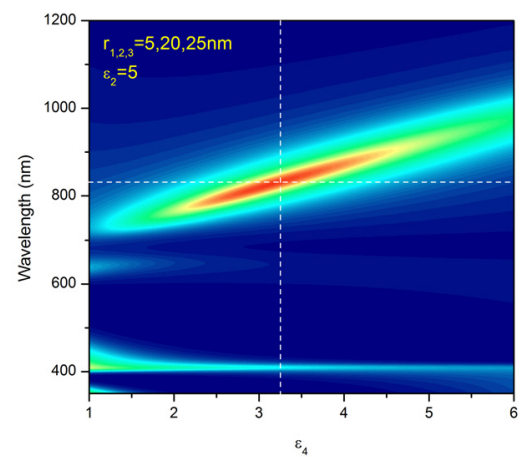

(a)

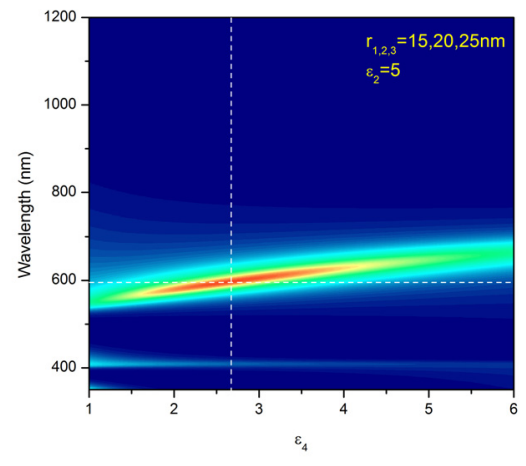

(c)
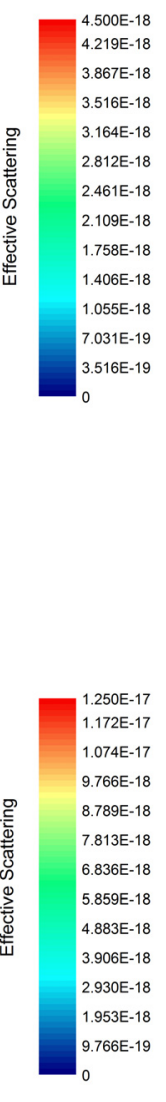

Figure 3 The effect of $\varepsilon_{4}$ on the effective scattering spectra of Au-Di-Ag nanoshells. (a) with a small inner gold nanosphere and large middle layer dielectric constant, (b) with a small inner gold nanosphere and small middle layer dielectric constant, (c) with a large inner gold nanosphere and large middle layer dielectric constant, (d) with a large inner gold nanosphere and small middle layer dielectric constant.

\subsubsection{The effect of inner core size on the effective scattering}

In order to illuminate the inner sphere radius-induced SPR transformation between $\left|\omega_{-}^{-}\right\rangle$and $\left|\omega_{-}^{+}\right\rangle$mode, we studied the effect of $r_{l}$ on the effective scattering of the Au@Ag bimetal three-layered nanostructure in this section. Figure 4 summarized the effect of $r_{l}$ on the effective scattering spectra of Au-dielectric-Ag nanoshells 
with different outer shell thickness and local dielectric environment. In Figure 4a,b,c, the local environment has the dielectric constant of $\varepsilon_{2,4}=5,2$, i.e., the separate layer has a larger dielectric constant than that of outer surrounding medium. It has been found that the increase of $r_{1}$ always leads to the effective scattering of $\left|\omega_{-}^{-}\right\rangle$ mode decrease, whereas the $\left|\omega_{-}^{+}\right\rangle$mode gets intense. When the outer silver shell has a thin thickness of $3 \mathrm{~nm}$, the effective scattering of $\left|\omega_{-}^{-}\right\rangle$mode is very weak, and only the $\left|\omega_{-}^{+}\right\rangle$mode takes the major role in the spectra. The inset of Figure 4a shows that the effective scattering peak of $\left|\omega_{-}^{+}\right\rangle$mode gets intense quickly as the $r_{l}$ is increased from 10 to $20 \mathrm{~nm}$. When the outer silver shell has a thickness of $9 \mathrm{~nm}$, the effective scattering of $\left|\omega_{-}^{-}\right\rangle$mode becomes greater and blue shifts to approach the $\left|\omega_{-}^{+}\right\rangle$mode. Thus the two effective scattering peaks begin to blend and overlap. Therefore, the decrease of the $\left|\omega_{-}^{-}\right\rangle$mode and the increase of the $\left|\omega_{-}^{+}\right\rangle$ mode results in the effective scattering changes non-monotonously. The inset of Figure $4 \mathrm{~b}$ shows that the effective scattering peak decreases firstly and reaches the minimum when $r_{l}=7 \mathrm{~nm}$, and then gets intense quickly as the $r_{l}$ is further increased. In Figure 4c, the outer silver shell has a large thickness of $15 \mathrm{~nm}$, which leads to the $\left|\omega_{-}^{-}\right\rangle$ mode further increases and blue shifts. Thus the two effective scattering peaks blend together completely. However, the $r_{1}$-dependent non-monotonous intensity change becomes slight, as shown in the inset of Figure 4c. In Figure $4 \mathrm{~d}, \mathrm{e}, \mathrm{f}$, the local environment has the dielectric constant of $\varepsilon_{2,4}=2,5$, i.e., the separate layer has a smaller dielectric constant than that of outer surrounding medium. In this case, the effective scattering of $\left|\omega_{-}^{-}\right\rangle$and $\left|\omega_{-}^{+}\right\rangle$mode always take the major role in the spectra. As the radius of the inner sphere is increased, the effective scattering of $\left|\omega_{-}^{-}\right\rangle$mode decreases, but the $\left|\omega_{-}^{+}\right\rangle$mode gets intense, which results in distinct SPR transformation from $\left|\omega_{-}^{-}\right\rangle$to $\left|\omega_{-}^{+}\right\rangle$mode. The comparison in Figure 4 also indicates that the most intense effective scattering could be obtained in the Au@Ag three-layered nanostructure with a thick outer Ag shell. Especially when the separate nanolayer has a larger dielectric constant, the intense effective scattering could be always obtained with different radius of the inner Au nanosphere. 


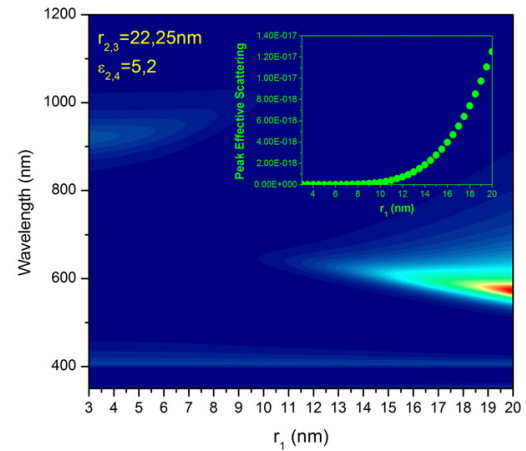

(a)

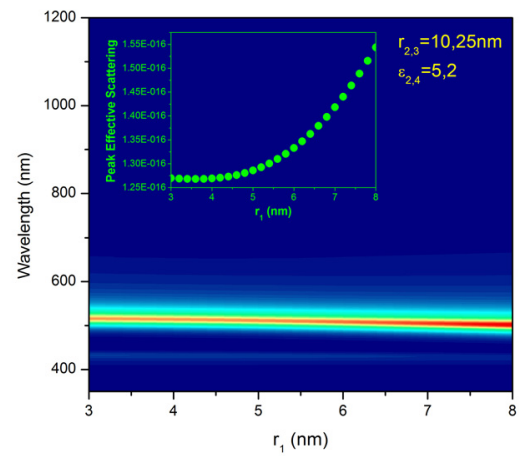

(c)

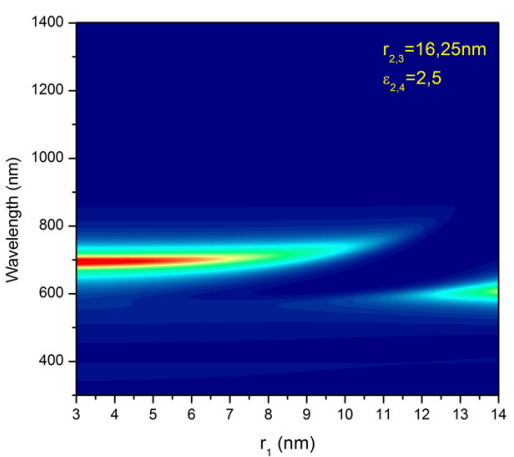

(e)
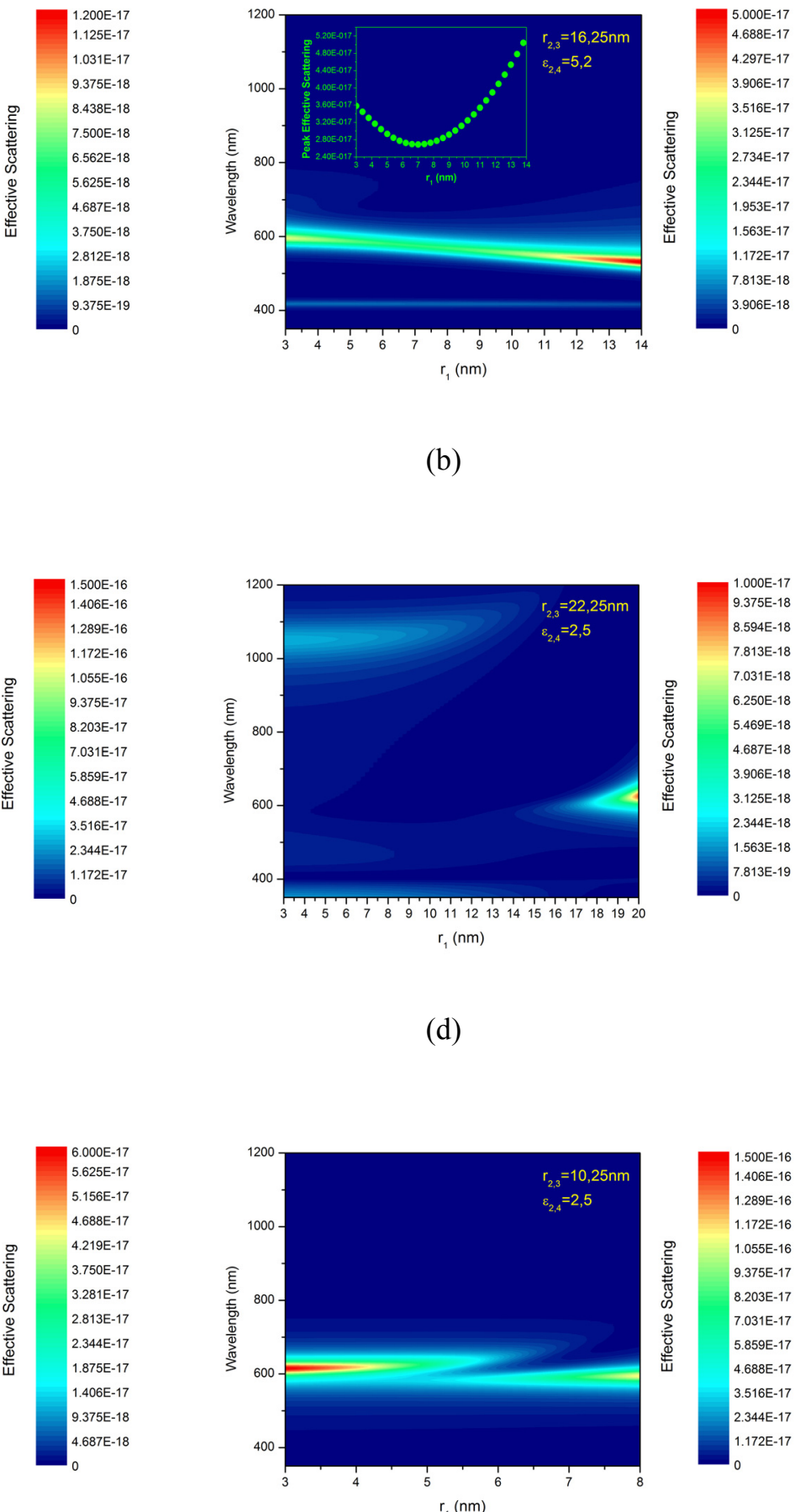

(f)

Figure 4 The effect of $r_{1}$ on the effective scattering spectra of Au-Di-Ag nanoshells. (a) with a thin thickness of the $\mathrm{Ag}$ shell and $\varepsilon_{2}>\varepsilon_{4}$, (b) with a middle thickness of the Ag shell and $\varepsilon_{2}>\varepsilon_{4}$, (c) with a thick thickness of the Ag shell and $\varepsilon_{2}>\varepsilon_{4}$, (d) with a thin thickness of the Ag shell and $\varepsilon_{2}<\varepsilon_{4}$, (e) with a middle thickness of the Ag shell and $\varepsilon_{2}<\varepsilon_{4}$,

(f) with a thick thickness of the Ag shell and $\varepsilon_{2}<\varepsilon_{4}$. 


\section{Conclusions}

The effective scattering of Au-dielectric-Ag three-layered nanoshells could be enhanced by tuning the geometric dimension and dielectric constant of local environment. The maximum effective scattering takes place when the outer Ag nanoshell has a thick thickness or the separate layer has a small dielectric constant. The effective scattering is also dependent on the radius of the inner Au sphere and the dielectric constant of the outer surrounding medium. Because of the plasmon mode transformation between $\left|\omega_{-}^{-}\right\rangle$and $\left|\omega_{-}^{+}\right\rangle$, the maximum effective scattering usually takes place when the inner Au sphere has a small or large radius. The increase of the surrounding dielectric constant leads to the effective scattering get intense firstly and then decreases. Therefore, the maximum effective scattering could be obtained when the surrounding dielectric constant has an intermediate value. These calculating results about effective plasmonic scattering of Au@Ag three-layered nanoshells presents a potential for design and fabrication of plasmonic optical nanodevice based on light scattering.

\section{Acknowledgements}

This work was supported by the National Natural Science Foundation of China under grant No. 11774283 


\section{Declarations:}

-Funding

This work was supported by the National Natural Science Foundation of China under grant No. 11774283

(Recipient: Jian Zhu)

-Competing Interests

The authors declare that they have no known competing financial interests or personal relationships that could have appeared to influence the work reported in this paper.

- Availability of data and material

Not applicable

- Code availability

Not applicable

-Authors Contributions

Jian Zhu: Writing-Reviewing and Editing, Investigation, Project administration, Funding acquisition.

Shu-min Zhao: Data curation, Investigation, Writing- Original draft preparation.

-Ethical Approval

Not applicable

-Consent to Participate

Not applicable

-Consent to Publish

The work described has not been published before.

The work is not under consideration for publication elsewhere.

Its publication has been approved by all co-authors.

Its publication has been approved by the responsible authorities at the institution where the work is carried out. 


\section{References}

[1] S.K. Eah, H.M. Jaeger, N.F. Scherer, Plasmon scattering from a single gold nanoparticle collected through an optical fiber. Appl. Phys. Lett. 86, 031902 (2005)

[2] S.W. Chu, T.Y. Su, R. Oketani, Y.T. Huang, H.Y. Wu, Y. Yonemaru, M. Yamanaka, H. Lee, G.Y. Zhuo, M.Y. Lee, S. Kawata, K. Fujita, Measurement of a Saturated Emission of Optical Radiation from Gold Nanoparticles: Application to an Ultrahigh Resolution Microscope. Phys. Rev. Lett. 112, 017402 (2014)

[3] M.R. Singh, M. C. Sekhar, S. Balakrishnan, S. Masood, Medical applications of hybrids made from quantum emitter and metallic nanoshell, J. Appl. Phys. 122, 034306 (2017)

[4] B.S. Luk'yanchuk, C.W. Qiu, Enhanced scattering efficiencies in spherical particles with weakly dissipating anisotropic materials. Appl. Phys. A 92, 773-776 (2008)

[5] H. Wang, P. Liu, Y. Ke, Y. Su, L. Zhang, N. Xu, S. Deng, H. Chen, Janus Magneto-Electric Nanosphere Dimers Exhibiting Unidirectional Visible Light Scattering and Strong Electromagnetic Field Enhancement. ACS Nano 9, 436-448 (2015)

[6] H. Oh, Y. Park, H. Song, Tracking Underpotential Deposition of Copper on Individual Silver Nanocubes by Real-Time Single-Particle Plasmon Scattering Imaging, J. Phys. Chem. C 124, 20398-20409 (2020)

[7] Q. Li, Z.H. Shi, L.J. Wu, H. Wei, Resonant scattering-enhanced photothermal microscopy, Nanoscale 12, $8397-8403(2020)$

[8] Y.B. Liu, T.T. Zhai, Y.Y. Liang, Y.B. Wang, X.H. Xia, Gold core-satellite nanostructure linked by oligonucleotides for detection of glutathione with LSPR scattering spectrum, Talanta 193, 123-127 (2019)

[9] C.T. Yang, M. Pourhassan-Moghaddam, D.P. Tran,L. Wu, X. Zhou, B. Thierry, Surface Plasmon Enhanced Light Scattering Biosensing: Size Dependence on the Gold Nanoparticle Tag, Sensors, 19, 323 (2019)

[10] J. Zhu, J.J. Li, J.W. Zhao, Tuning the wavelength drift between resonance light absorption and scattering of plasmonic nanoparticle. Appl. Phys. Lett. 99, 101901 (2011)

[11] K.S. Lee, M.A. El-Sayed, Dependence of the Enhanced Optical Scattering Efficiency Relative to That of Absorption for Gold Metal Nanorods on Aspect Ratio, Size, End-Cap Shape, and Medium Refractive Index. J. Phys. Chem. B 109, 20331-20338 (2005)

[12] V.A. Dhumale, P.V. Shah, R.B. Sharma, K. Tanabe, Effects of particle size and surrounding media on optical radiation efficiencies of spherical plasmonic metal nanoparticles. Bull. Mater. Sci. 35, 143-149 (2012)

[13] K. Park, S. Biswas, S. Kanel, D. Nepal, R.A. Vaia, Engineering the Optical Properties of Gold Nanorods: Independent Tuning of Surface Plasmon Energy, Extinction Coefficient, and Scattering Cross Section. J. Phys. 
Chem. C 118, 5918-5926 (2014)

[14] A. Bansal, J.S. Sekhon, S.S. Verma, Scattering Efficiency and LSPR Tunability of Bimetallic Ag, Au, and Cu Nanoparticles. Plasmonics 9, 143-150 (2014)

[15] A. Bansal, S.S. Verma, Tailoring LSPR-Based Absorption and Scattering Efficiencies of Semiconductor-Coated Au nanoshells. Plasmonics 9, 335-341 (2014)

[16] M.R Singh, P.D. Persaud1, S. Yastrebov, A study of two-photon florescence in metallic nanoshells, Nanotechnology 31, 265203 (2020)

[17] J. Qian, Y. Li, J. Chen, J. Xu, Q. Sun, Localized Hybrid Plasmon Modes Reversion in Gold-Silica-Gold Multilayer Nanoshells. J. Phys. Chem. C 118, 8581-8587 (2014)

[18] M.R Singh, S. Yastrebov, Switching and Sensing Using Kerr Nonlinearity in Quantum Dots Doped in Metallic Nanoshells, J. Phys. Chem. C 124, 12065-12074 (2020)

[19] C. Liu, J. Lv, Z. Liu, S. Zheng, Q. Liu, T. Sun, H. Mu, P.K. Chu, Theoretical Assessment of Localized Surface Plasmon Resonance Properties of Au-Interlayer-Ag Multilayered Nanoshells, Plasmonics 11, 1589-1595 (2016)

[20] D.J. Wu, X.W. Wu, Y. Cheng, B.B. Jin, X.J. Liu, Dual-frequency plasmon lasing modes in active three-layered bimetallic Ag/Au nanoshells, Appl. Phys. Lett. 107, 191909 (2015)

[21] Y. Gao, Y. Li, Y. Wang, Y. Chen, J. Gu, W. Zhao, J. Ding, J. Shi, Controlled Synthesis of Multilayered Gold Nanoshells for Enhanced Photothermal Therapy and SERS Detection, Small 11, 77-83 (2015)

[22] J. Zhu, Z.J. Xu, G.J. Weng, J. Zhao, J.J. Li, J.W. Zhao, Etching-dependent fluorescence quenching of Ag-dielectric-Au three-layered nanoshells: The effect of inner Ag nanosphere, Spectrochimica Acta Part A: Molecular and Biomolecular Spectroscopy, 200, 43-50 (2018)

[23] D.E. Ponton, M.N. Croteau, S.N. Luoma, S. Pourhoseini, R.C. Merrifield, J.R. Lead, Three-layered silver nanoparticles to trace dissolution and association to a green alga, Nanotoxicology 13, 1149-1160 (2019)

[24] D.J. Wu, X.D. Xu, X.J. Liu, Influence of dielectric core, embedding medium and size on the optical properties of gold nanoshells. Solid State Commun. 146, 7-11 (2008)

[25] J. Zhu, S.M. Zhao, The Effect of Metal Type on the Separate Dielectric Layer-Dependent Plasmon Coupling in Al-Au Bimetallic Metal-Dielectric-Metal Nanoshells. Plasmonics 10, 297-304 (2015)

[26] J. Zhu, J.J. Li, J.W. Zhao, Local Dielectric Environment Dependent Local Electric Field Enhancement in Double Concentric Silver Nanotubes. J. Phys. Chem. C 117, 584-592 (2013)

[27] J. Zhu, J.J. Li, J.W. Zhao, The Effect of Dielectric Coating on the Local Electric Field Enhancement of 
Au-Ag Core-Shell Nanoparticles. Plasmonics 10, 1-8 (2015)

[28] J.A.A.J. Perenboom, P. Wyder, F. Meier, Electronic properties of small metallic particles. Phys. Rep. 78, 173-292 (1981)

[29] J. Zhu, Y.J. Ren, S.M. Zhao, J.W. Zhao, The effect of inserted gold nanosphere on the local field enhancement of gold nanoshell. Mater. Chem. Phys. 133, 1060-1065 (2012)

[30] J.W. Haus, H.S. Zhou, S. Takami, M. Hirasawa, I. Honma, H. Komiyama, Enhanced optical properties of metal-coated nanoparticles. J. Appl. Phys. 73, 1043-1048 (1993)

[31] J. Zhu, J.J. Li, J.W. Zhao, S.W. Bai, Light absorption efficiencies of gold nanoellipsoid at different resonance frequency. J. Mater. Sci. 43, 5199-5205 (2008)

[32] Y.W. Ma, L.H. Zhang, Z.W. Wu, J.C. You, X.C. Yin, J. Zhang, G.S. Jian, Theoretical Studies of Tunable Localized Surface Plasmon Resonance of Gold-Dielectric Multilayered Nanoshells, Plasmonics 12, 1057-1070 (2017) 
Figures

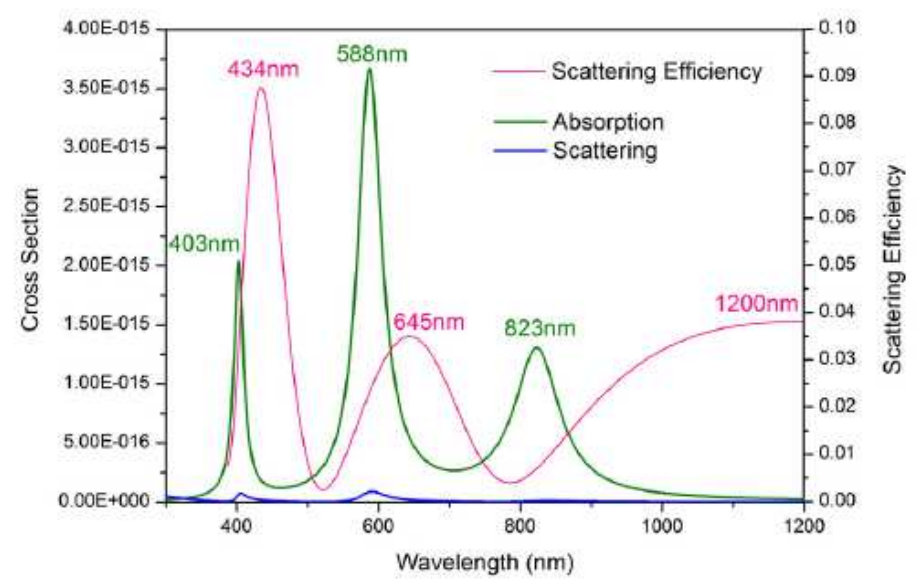

(a)

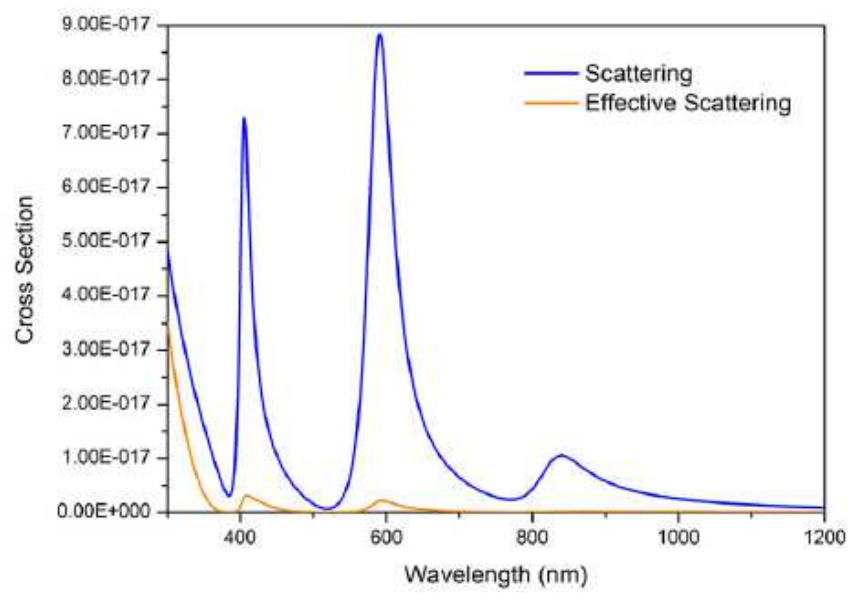

(b)

\section{Figure 1}

(a) The spectrum of light absorption, scattering and scattering efficiency. (b) The spectral comparison between scattering and effective scattering. $r 1,2,3=[10,20,25] \mathrm{nm}, \varepsilon 2,4=[5,1]$. 


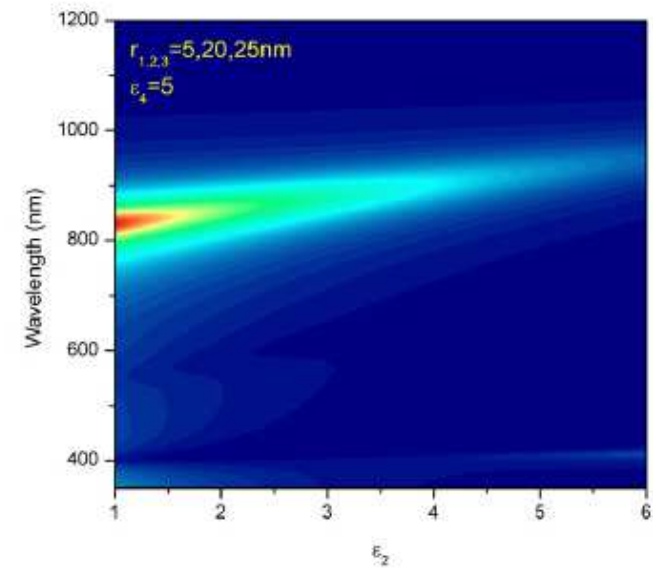

(a)

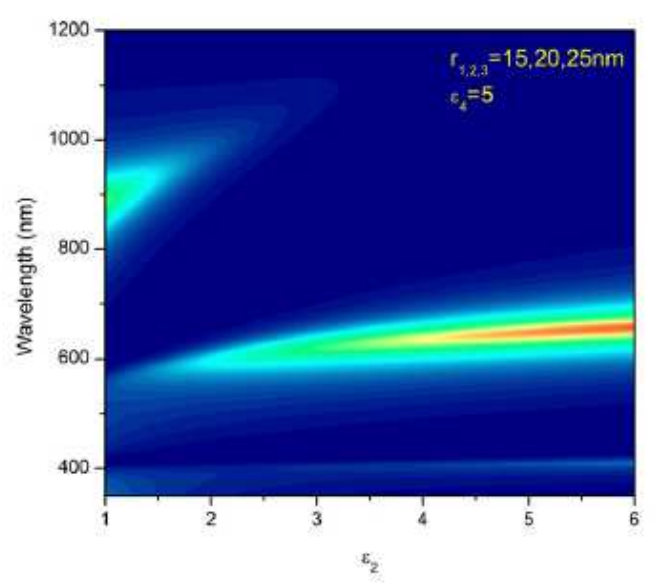

(c)
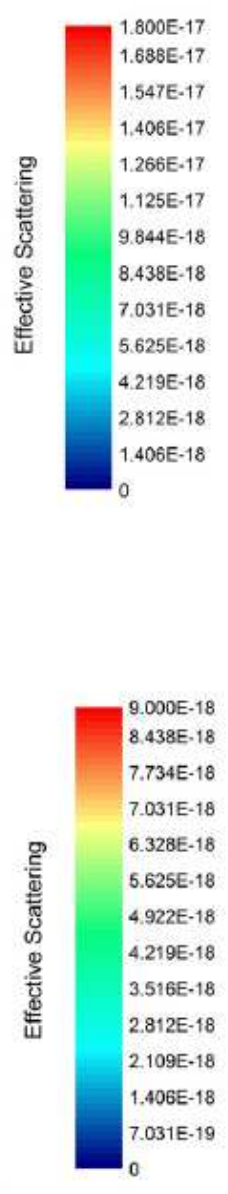

Figure 2

The effect of $₫ 2$ on the effective scattering spectra of Au-Di-Ag nanoshells. (a) with a small inner gold nanosphere and large environmental dielectric constant, (b) with a small inner gold nanosphere and small environmental dielectric constant, (c) with a large inner gold nanosphere and large environmental dielectric constant, (d) with a large inner gold nanosphere and small environmental dielectric constant. 


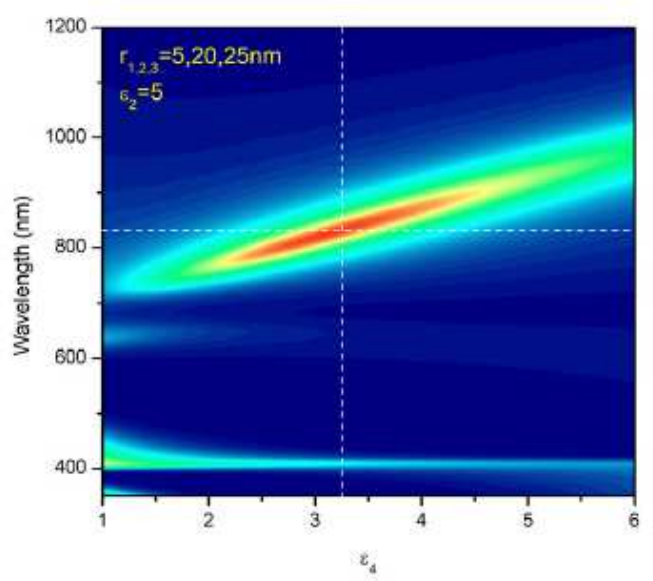

(a)

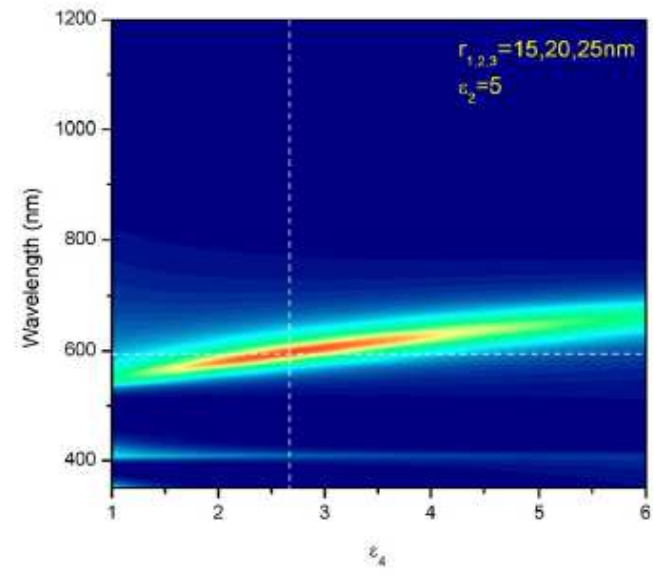

(c)
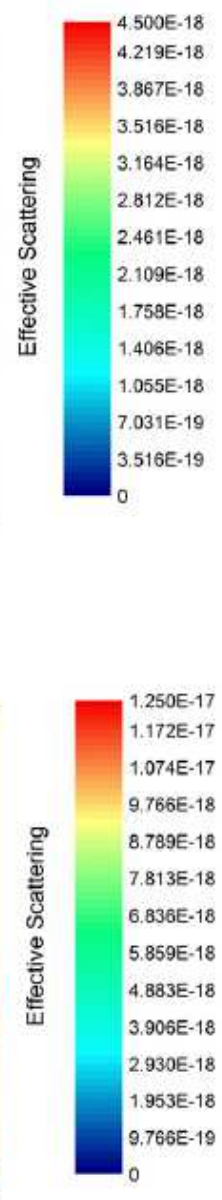

\section{Figure 3}

The effect of $₫ 4$ on the effective scattering spectra of Au-Di-Ag nanoshells. (a) with a small inner gold nanosphere and large middle layer dielectric constant, (b) with a small inner gold nanosphere and small middle layer dielectric constant, (c) with a large inner gold nanosphere and large middle layer dielectric constant, (d) with a large inner gold nanosphere and small middle layer dielectric constant.

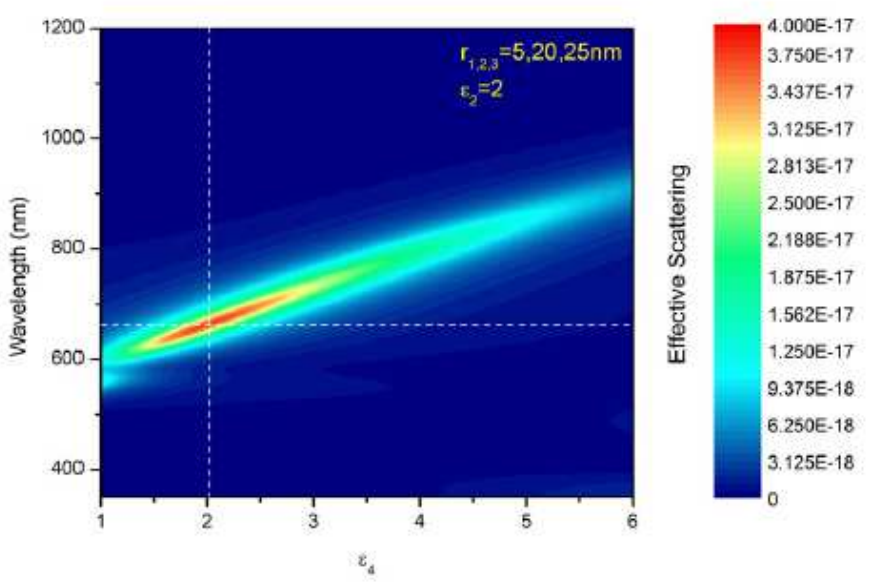

(b)

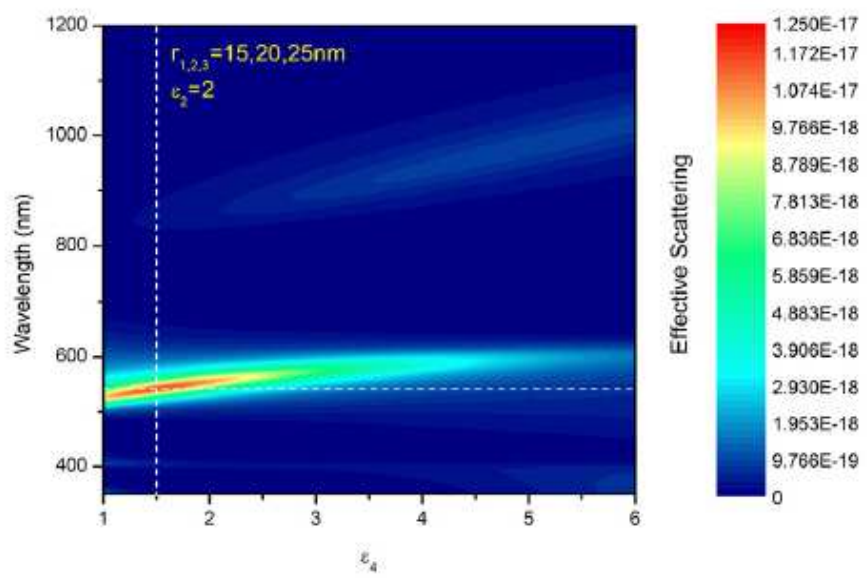

(d) 


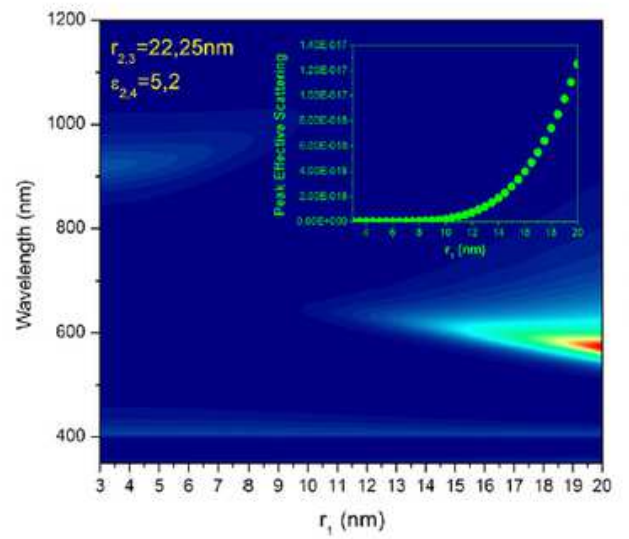

(a)

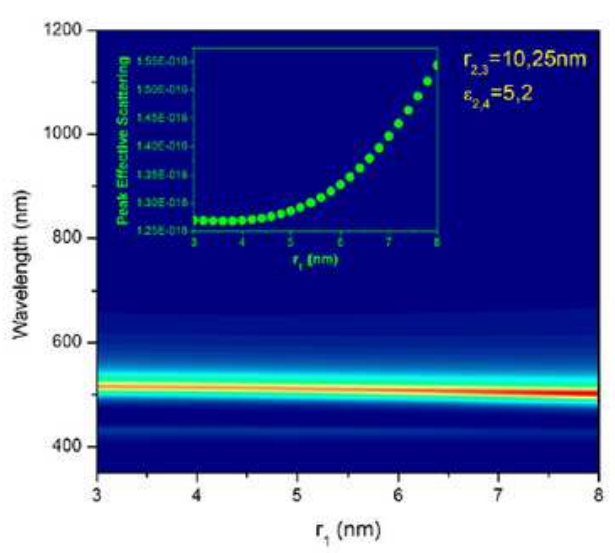

(c)

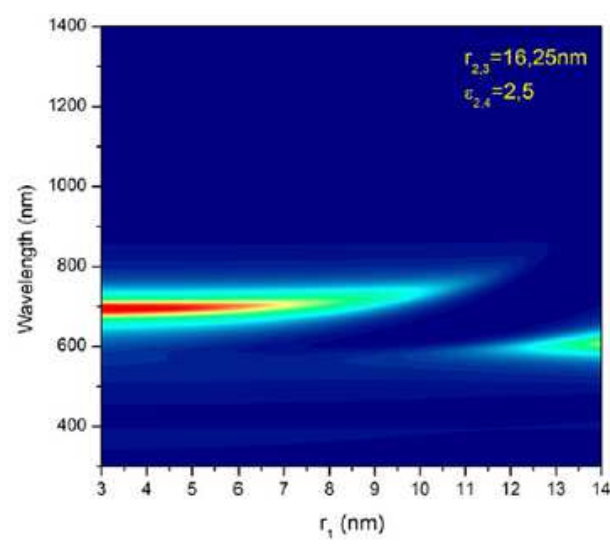

(e)
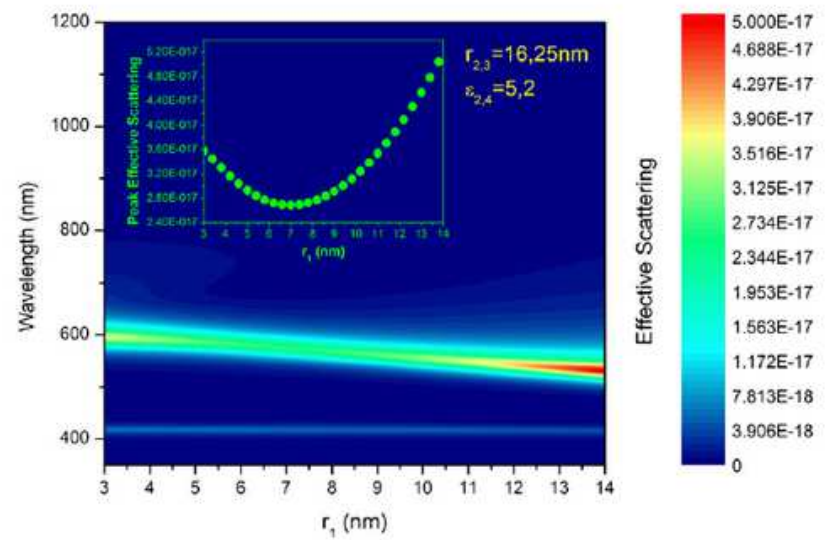

(b)
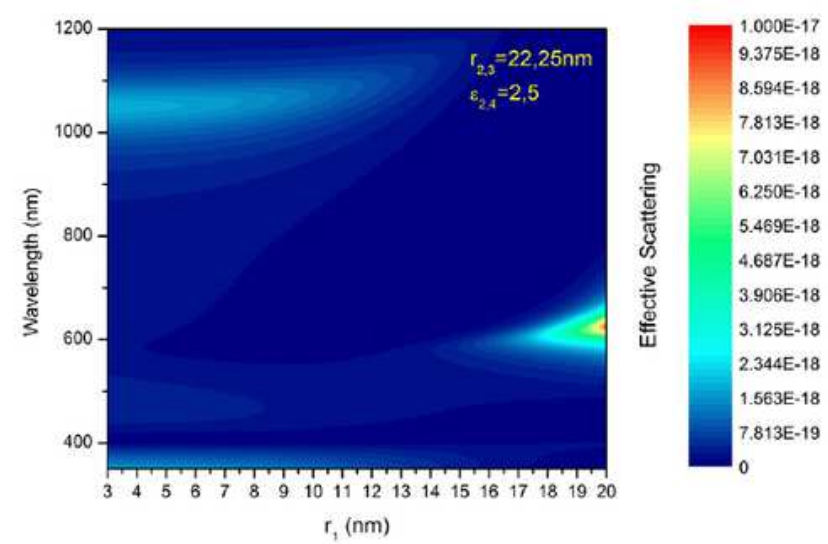

(d)

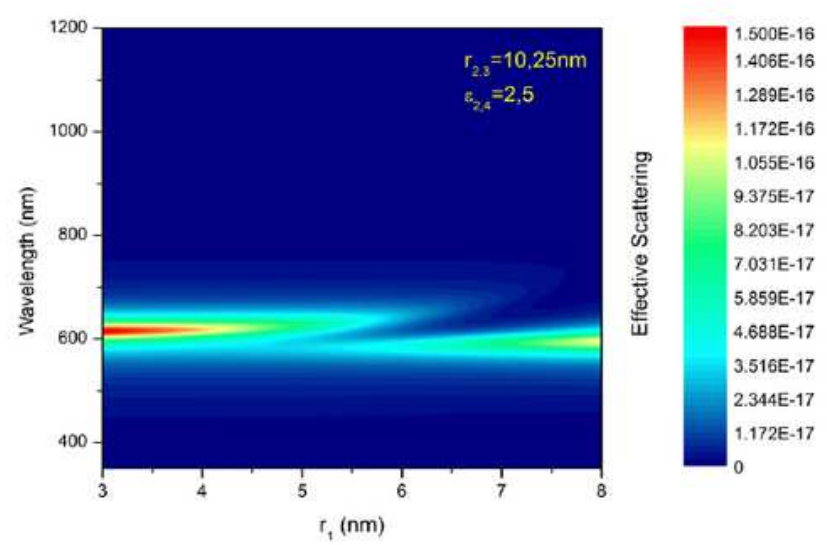

(f)

\section{Figure 4}

The effect of $r 1$ on the effective scattering spectra of Au-Di-Ag nanoshells. (a) with a thin thickness of the $\mathrm{Ag}$ shell and $\varepsilon 2>\varepsilon 4$, (b) with a middle thickness of the Ag shell and $\varepsilon 2>\varepsilon 4$, (c) with a thick thickness of the Ag shell and $\varepsilon 2>\varepsilon 4$, (d) with a thin thickness of the Ag shell and $\varepsilon 2<\varepsilon 4$, (e) with a middle thickness of the Ag shell and $\varepsilon 2<\varepsilon 4$, (f) with a thick thickness of the Ag shell and $\varepsilon 2<\varepsilon 4$. 